\title{
Fabrication Process and Performance Analysis of CSP LED Filaments with a Stacked Package Design
}

\author{
Nan Jiang ${ }^{1}$, Jun Zou ${ }^{1,2, *}$, Changran Zheng ${ }^{3}$, Mingming Shi ${ }^{2}$, Wenbo Li ${ }^{4}$, Yiming Liu ${ }^{1}$, \\ Bin Guo ${ }^{3}$, Jerry Liu ${ }^{5}$, Herry Liu ${ }^{5}$ and Xavier Yin ${ }^{5}$ \\ 1 School of Meterial Scince and Engineering, Shanghai Institute of Technoligy, Shanghai 201418, China; \\ 166081101@mail.sit.edu.cn (N.J.); 166081116@mail.sit.edu.cn (Y.L.) \\ 2 School of Science, Shanghai Institute of Technology, Shanghai 201418, China; mmshi@sit.edu.cn \\ 3 School of Material Science and Engineering, Changchun University of Science and Technology, \\ Changchun 130022, China; 1228746314@qq.com (C.Z.); guobin@cust.edu.cn (B.G.) \\ 4 Zhejiang Emitting Optoelectronic Technology Company, Zhejiang 310000, China; liwenbo@emitting.cn \\ 5 Tecore Synchem, INC., Tianjin 300451, China; Jerry_liu@tecore-synchem.com (J.L.); \\ Herry_liu@tecore-synchem.com (H.L.); Xavier_yin@tecore-synchem.com (X.Y.) \\ * Correspondence: zoujun@sit.edu.cn
}

Received: 25 September 2018; Accepted: 8 October 2018; Published: 16 October 2018

check for updates

\begin{abstract}
This article presents a chip-scale package (CSP) based filament light-emitting diodes (LEDs) with a stacked-type structure and a segmented-type luminescence design for higher luminous efficiency and better electrical stability. The innovation strategy improves the luminous efficiency by $7.69 \%$ and $18.97 \%$ than the traditional dispensing packaged LED filament when the current is at $30 \mathrm{~mA}$ and $210 \mathrm{~mA}$, respectively, and reduces the use of red phosphors and green phosphors by $66.7 \%$ and $26.2 \%$, respectively. With the increase of the current, the luminous efficiency variations of the stacked-packaged filament are lower than that of the traditional dispensing packaged filament. Moreover, the color temperature of the filament is no longer determined by the calculation of the ratio of various phosphors. The stacked structure and the segmented design can help us to adjust the specified color temperature quickly by changing the proportion and position of the blue chips and the red CSP LEDs.
\end{abstract}

Keywords: optical design and fabrication; chip-scale package; light-emitting diode; optical devices

\section{Introdution}

Attributed to their high efficiency, long life, low power consumption, environmental friendliness, tiny size, and directionality, the recently-developed phosphor-converted white light-emitting diode (WLED) have become the most promising substitute to conventional light sources [1-4]. However, there are still many problems, including the improvement of phosphor conversion and luminous efficiency [5], and improvements in color purity for their substitution of cheaper incandescent and fluorescent lamps that currently occupy the global lighting market [6].

Presently, light-emitting diode (LED) filaments are a fervent LED product. They have three-dimensional luminescence and high plasticity, and can control light distribution according to the application environment. In contrast, the shortcomings are also very prominent, such as low color purity, low light efficiency, and low heat dissipation, so it is difficult to meet the standards of indoor lighting. The dispensing method of traditional LED filament packaging technology has a large variation in optical performance and the drift of color temperature under different currents $[7,8]$. Color purity is the key to improving luminous efficiency and light output. The blue InGaN LED chip pumped by blue/green phosphor is a mature technology; however, these lack red components [9]. 
Therefore, the mixing of a red phosphor viz. $\mathrm{Sr}_{2} \mathrm{Si}_{5} \mathrm{~N}_{8}$ : Eu ${ }^{2+}$ and green/yellow phosphor, namely, yttrium aluminum garnet (YAG): $\mathrm{Ce}^{3+}$ or $\mathrm{SrSi}_{2} \mathrm{O}_{2} \mathrm{~N}_{2}: \mathrm{Eu}^{2+}$, is one method to overcome the problem of impure color. Nevertheless, low color purity can be caused by spectral overlapping, which happens when the partial reabsorption of emitted light by another phosphor, mixed with the design of the two phosphors, occurs. [10-15]. To tackle this problem, stacked phosphor layers were developed by stacking green and red phosphor layers, which have been found to have higher efficiencies. This design showed that the reabsorption of green emissions by red components is reduced, when a red phosphor is used under the green phosphor layer [16].

However, thus far, most of the research on stacked package structures is focused on remote type packages, such as phosphor in glass (PiG), fluorescent film, and other forms $[17,18]$. For example, Xiang et al. [19] found that screen-printing a red phosphor layer on LuAG:Ce ${ }^{3+}$ PiG obtained a thermally-stable warm WLED. Lee et al. [20] designed a new phosphor design for reducing the spectral overlapping of PiGs, by cutting and reassembling commercial green $\mathrm{Lu}_{3} \mathrm{Al}_{5} \mathrm{O}_{12}: \mathrm{Ce}^{3+}\left(\mathrm{LuAG} \mathrm{Ce}^{3+}\right)$ and red $\mathrm{CaAlSiN}_{3}: \mathrm{Eu}^{2+}\left(\mathrm{CASN}: \mathrm{Eu}^{+}\right)$PiGs. Inspired by what has been explained above, our team planned to solve the performance problem of LED filaments by combining chip scale package (CSP) with a traditional dispensing method. In recent years, CSP has become a very popular research topic in the LED packaging field [21,22]. The package size is only slightly larger than an LED chip, with an area ratio of 1.5:1 [23]. CSP has the advantages of having a small package size and high assembly density [21]. Thus, in this work, we report a new phosphor design for reducing the spectral overlapping of LED filaments by using a CSP layer and a layer of dispensing phosphor.

\section{Experimental Section}

The fluorescent film used for packaging is made by TECORE SYNCHEM, Inc., Tianjin, China, our partner company, using their self-developed preparing technology. A red-emitting phosphor CASN: $\mathrm{Eu}^{2+}$, with a wavelength of $618 \mathrm{~nm}$, was used to make the fluorescent film with a mass fraction of $22.2 \mathrm{wt} . \%$. The manufacturing process of CSP LEDs is shown in Figure 1. Firstly, under a certain pressure, rearranged chips were pressed into a softened film under vacuum conditions. Then, after being baked in an oven for three hours at a temperature of $300^{\circ} \mathrm{C}$, the pressed chips coated with film were cut into pieces, which we called CSP LEDs. After that, the red CSP LEDs were welded to a 135-mm long flexible substrate using a solid crystal machine. Each flexible filament substrate was welded with 51 CSP chips, which were connected in series. The uniform mixture of green-emitting phosphor LuAG:Ce ${ }^{3+}$ and package resin was used as a second layer package for the CSP filament. Compared with the traditional dispensing packaging method, this packaging method is more complex but has a lower cost.

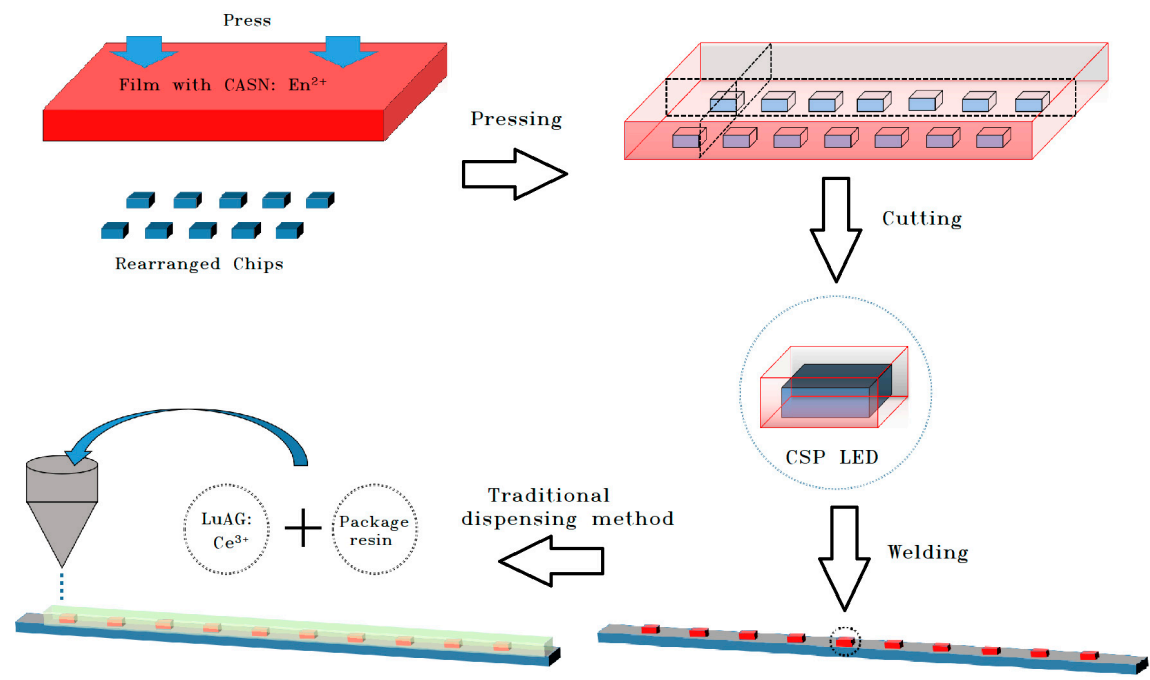

Figure 1. Schematic stacked type chip-scale package (CSP) filament design and fabrication. 
As mentioned above, stacked packaging structure can improve the optical efficiency of remote encapsulation of an LED. However, due to the air gap between the interfaces of the two packages, there are many optical phenomena, such as refraction, reflection and scattering at the interfaces [24]. Therefore, LEDs with stacked packages will not reach the ideal light effect, and the light effect will even be reduced due to the excessive absorption and barrier of red phosphor to blue light when the red phosphor layer is under the green phosphor layer. Therefore, a dry-wet separation package method, on the basis of a stacked packaging structure, was adopted to eliminate the air gap by the wet layer wrapping the dry layer completely.

As we know, red-emitting phosphors $\mathrm{CASN}: \mathrm{Eu}^{2+}$ have strong absorption and blocking effects on blue emissions [25]. The electro luminescence (EL) spectra obtained under the conditions of $30 \mathrm{~mA}$ and $140 \mathrm{~V}$, and the corresponding Commission Internationale de L'Eclairage-1931 (CIE-1931) chromaticity coordinates tested by the Yuan Fang integral ball under a condition of $30 \mathrm{~mA}$ and $140 \mathrm{~V}$, are shown in Figure 2a,b. The EL emission of the red CSP filament without the green package is composed of a blue emission at $450 \mathrm{~nm}$, and a broad emission that occurs at wavelengths ranging from 550 to $700 \mathrm{~nm}$; the former arises from the InGaN LED chip and the latter from the red CASN:Eu ${ }^{2+}$ phosphors. However, the EL emission of the red CSP filament with a green package includes little blue emission at $450 \mathrm{~nm}$, which means that the upper green phosphor almost completely absorbs the blue emissions emitted from the red CSP LEDs. With an increasing forward driving current, from 30 to $210 \mathrm{~mA}$, the corresponding CIE-1931 chromaticity coordinates are presented in Figure 2b. A comparison of Commission Internationale de L'Eclairage (CIE) color coordination reveals that the emitting lights of these two samples are all in red.
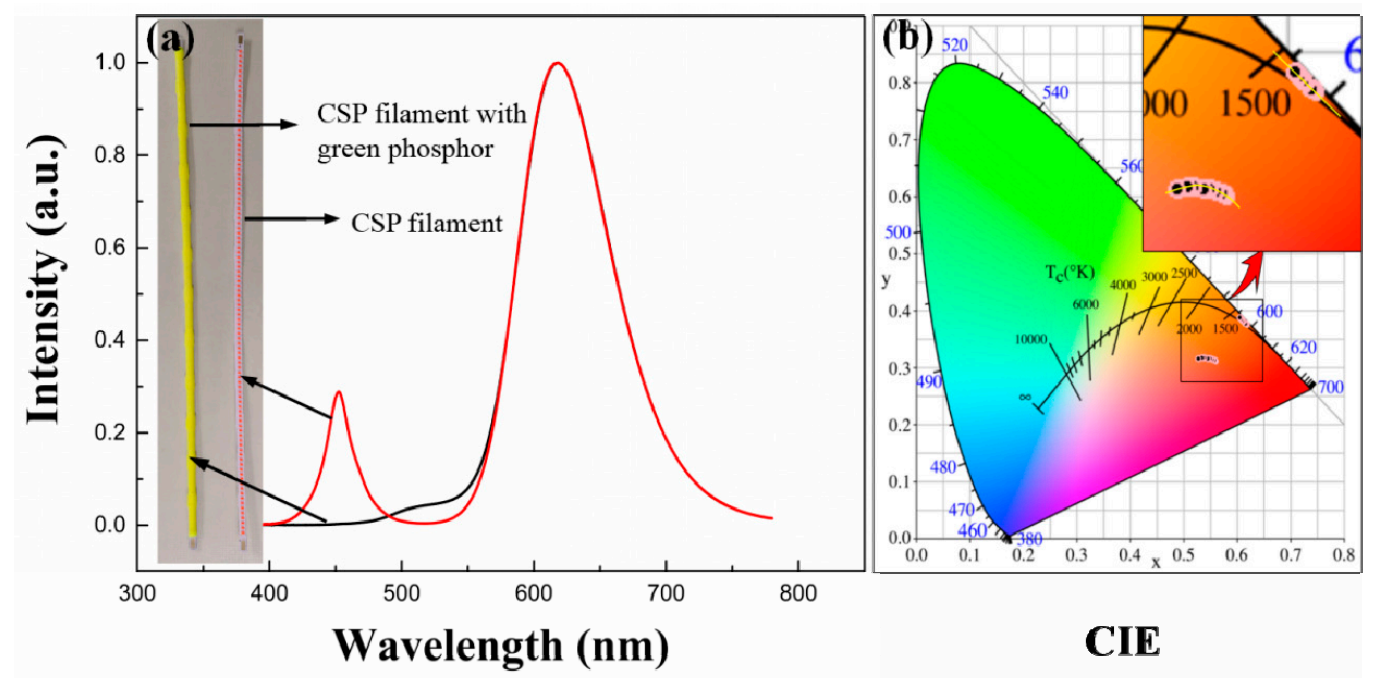

Figure 2. (a) Electro luminescence (EL) spectra of pure red CSP filament before and after package; (b) the Commission Internationale de L'Eclairage (CIE)-1931 chromaticity coordinates of pure red CSP filament before and after package.

In order to fully stimulate the red-emitting phosphors and let parts of the blue light overflow stimulate the green emitting phosphors, a small improvement was applied to the filament structure. As shown in Figure 3, blue chips were arranged regularly between red CSP LEDs, and the proportions of red CSP and blue light chips were 2:1, 1:1, 1:2 and 1:3, respectively. This package can be seen as a combination of stacked-type and segmented-type packages. The mass fraction of red CASN:Eu ${ }^{2+}$ in the film was determined, and the mass fraction of green LuAG:Ce ${ }^{3+}$ and the proportion of CSP and blue chips is controllable. Thus, luminous performance can be designed by controlling these two parameters. 


\section{Red:Blue}

2: 1

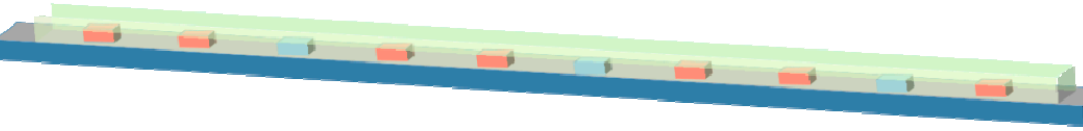

1: 1

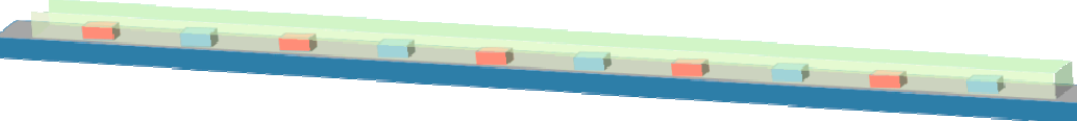

1: 2

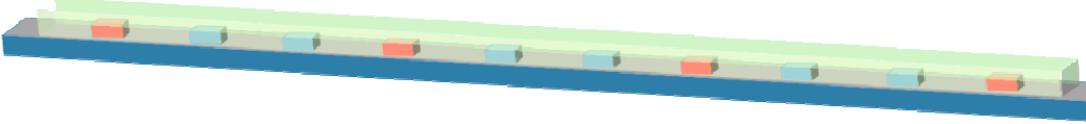

1: 3

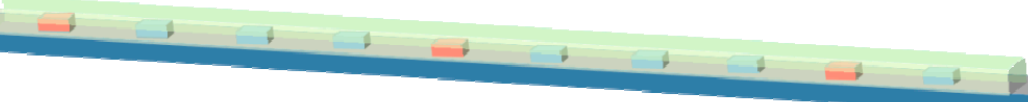

Figure 3. Samples with different chip proportions.

The four kinds of filaments mentioned above were dispensed with silicone resins in which the mass fractions of green LuAG:Ce ${ }^{3+}$ phosphor were $14.3 \%, 20 \%, 29.4 \%$. The CIE values obtained for the designed CSP LED filament samples, tested by the Yuan Fang integral ball under the conditions of $30 \mathrm{~mA}$ and $140 \mathrm{~V}$, are shown in Figure 4, from which we can see that the CIE color coordination has a wide range of distribution, and the correlated color temperatures can be obtained from $2200 \mathrm{~K}$ to $4600 \mathrm{~K}$. According to the requirements of indoor atmosphere lamps, samples with correlated color temperature (CCT) near $2500 \mathrm{~K}$ were used and compared with the traditional packaged LED filaments.

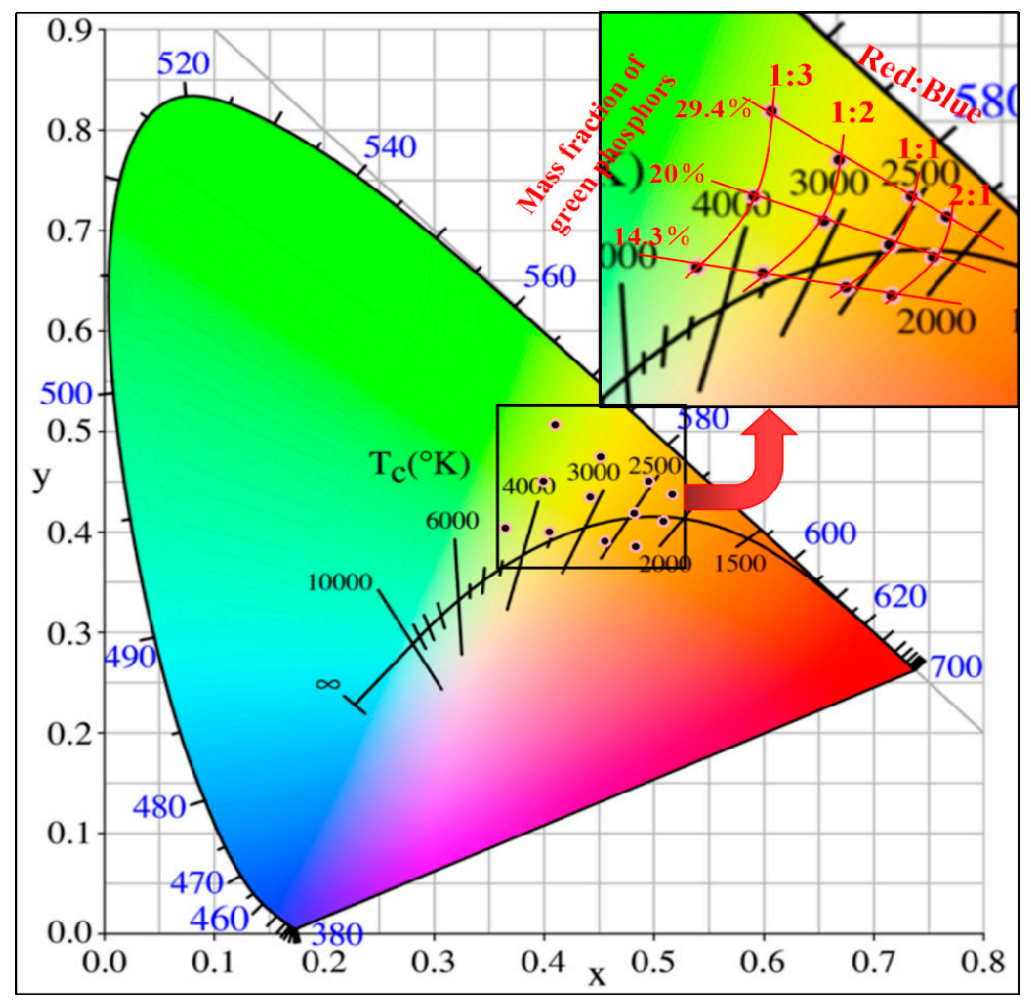

Figure 4. CIE values obtained for designed CSP LED filament samples. 


\section{Results and Discussion}

Figure 5 shows the practicality of two samples before and after the second-layer packaging. The first two samples were pure red CSP filaments, before and after the green LuAG:C $\mathrm{e}^{3+}$ packaging, respectively. The last sample was our designed filament, with a CCT of $2500 \mathrm{~K}$, which emits the warm white light, and the upper one is the sample before the designed $2500 \mathrm{~K}$ filament packaging. It can be seen that the colors of each sample's emitting light were different. Although the last filament in Figure 5 is a segmented-type luminescence design, the luminescence appeared very homogeneous. This phenomenon was due to the second layer of green packaging providing ample space for light reflection, diffusion and deflection in the silicone matrix. The CSP filament of the laminated package is shown in Figure 6, and the dimensions of each part can be seen clearly. The thickness of the green packaging layer was nearly three times that of the red film thickness, which was a favorable space for photon multiple conversions, so the filament could give uniform illumination.

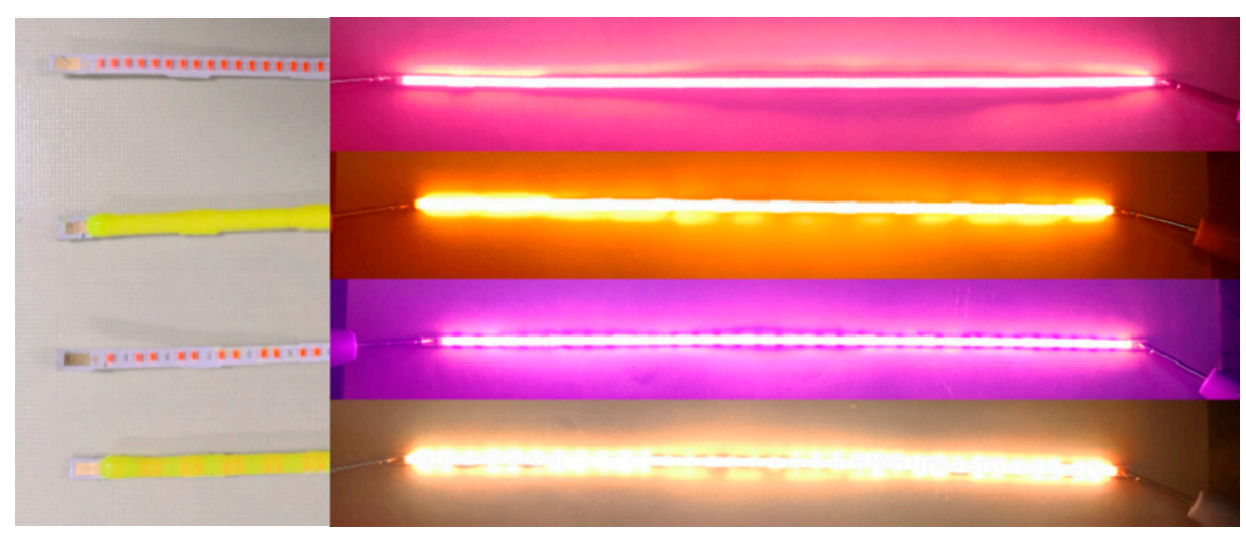

Figure 5. An actual image of the stacked type packaged filament.

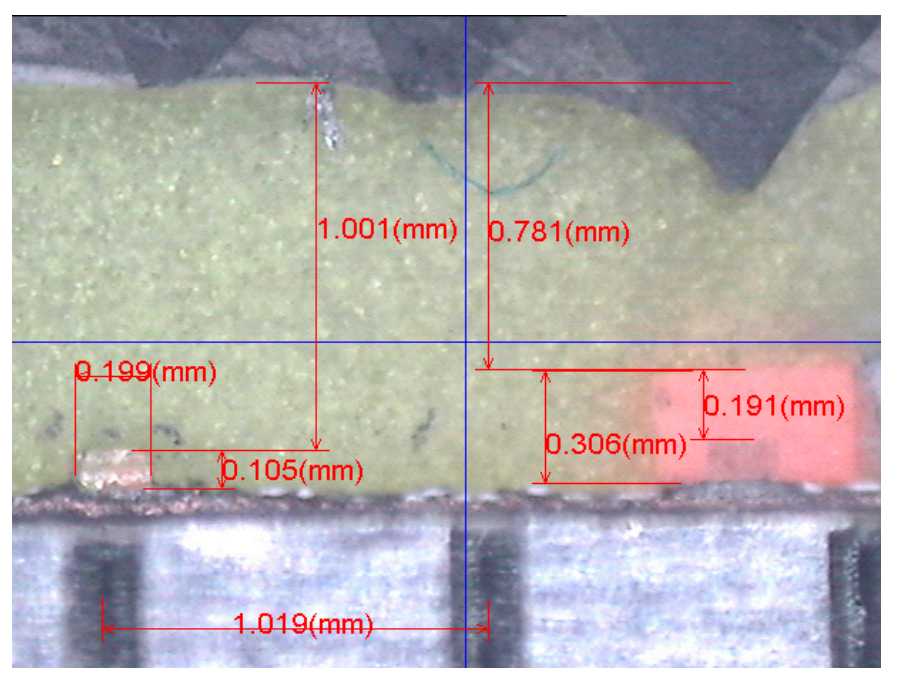

Figure 6. A microscopic image of stacked type packaged filament.

The EL emission of the stacked package and traditional dispensing package filaments, shown in Figure 7, were composed of a blue emission at $450 \mathrm{~nm}$, and a broad emission that occured at wavelengths ranging from 500 to $700 \mathrm{~nm}$, the former from the InGaN LED chip and the latter from the green LuAG:Ce ${ }^{3+}$ and red CASN:Eu ${ }^{2+}$ phosphors. The green EL emission intensity of the stacked-type packaged filament was found to be higher than the traditional dispensing packaged filament. This may be ascribed to the decreased absorption of the LuAG: $\mathrm{Ce}^{3+}$ s green emission by the red-emitting $\mathrm{CASN}: \mathrm{Eu}^{2+}$ phosphor, thereby increasing green emission intensity. These phenomena clearly indicated that spectral overlapping was effectively reduced using a stacked-type package and segmented-type 
luminescence design. The decrease in spectral overlapping observed in the current design of the CSP filament can be attributed to the successful organization of the proximity of the luminescent elements in the assembled CSP filaments. The interaction of these optical centers at an optimum distance promotes the spatial delocalization of energy, minimizes energy transfer between the luminescent centers and thereby reduces the spectral overlapping.

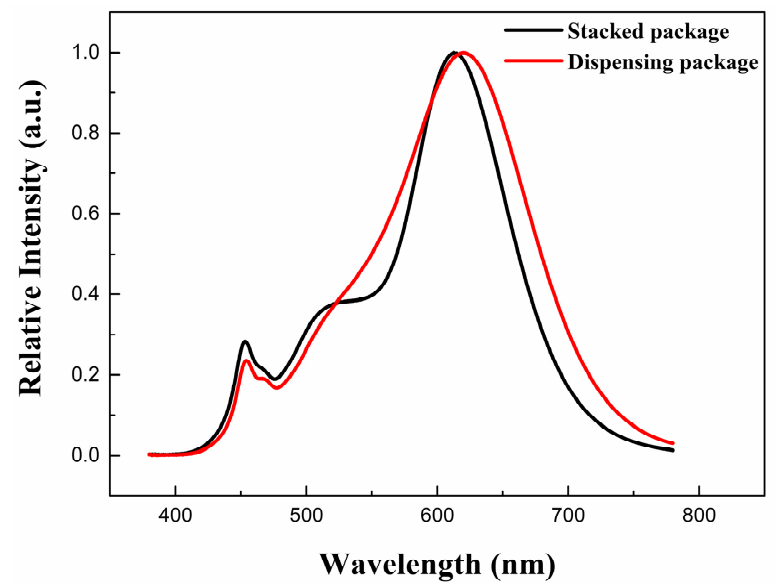

Figure 7. EL spectra of traditional dispensing packaged filament and stacked-type packaged filament.

With the same correlated color temperature of $2500 \mathrm{~K}$, the CIE color coordinates of the aforementioned filaments are shown in Figure 8. The CIE color coordinates of both samples move to the left, but with two different trajectories, while the current increases from 30 to $210 \mathrm{~mA}$. In the stacked-type filament, red fluorescent film can be considered to be in a state of excitation saturation; thus, with the increase in current, the emission of red light did not change obviously. Instead, the green phosphor stimulated more green light with an increasing intensity of blue light, so the color coordinates moved directly to the blue-green area. On the contrary, in the traditional dispensing package, phosphors were evenly dispersed in the silicone resins. As seen in Table 1, the luminous efficiency of the stacked-packaged filament was higher than that of the traditional dispensing packaged filament. With the increase in current, the luminous efficiency variations of the stacked-packaged filament were lower than that of the traditional dispensing packaged filament. This phenomenon can mainly be attributed to the reduction of photon reabsorption and the simplification of the photon transfer path.

Table 1. Electroluminescence data of traditional dispensing packaged filament and stacked-type packaged filament.

\begin{tabular}{ccc}
\hline & Traditional Dispensing Method & Stacked Packaging Method \\
\hline CCT at $30 \mathrm{~mA}(\mathrm{~K})$ & 2499 & 2506 \\
CRI at $30 \mathrm{~mA}(\mathrm{Ra})$ & 89.7 & 87.6 \\
CIE at $30 \mathrm{~mA}$ & $(0.4797,0.4180)$ & $(0.4797,0.4180)$ \\
Luminous efficiency at $30 \mathrm{~mA}(\mathrm{Lm} / \mathrm{W})$ & 68.62 & 73.88 \\
Luminous efficiency at $210 \mathrm{~mA}(\mathrm{Lm} / \mathrm{W})$ & 44.95 & 53.48 \\
The variations in luminous efficiency & 23.67 & 20.4 \\
The rate of variations in luminous efficiency & $34.94 \%$ & $27.61 \%$ \\
\hline
\end{tabular}

The general luminescence mechanism of Pc-White LEDs is the mixing of short wavelength light emitted by blue or UV LED chips and long-wavelength light emitted from phosphors pumped by the short wavelength light [26]. It is considered a complicated energy-conversion and optical tracing process when the input light interacts with a phosphor. With the law of conservation of energy, when a short wavelength light excites a phosphor, part of the energy is converted to long wavelength light and the other part is dissipated in the form of heat. As shown in Equation (F1), the input power from 
the blue LED chip $\left(E_{\text {input }}\right)$ can be transferred to the blue photon energy that transmits out of silicone $\left(E_{\text {transmitted }}\right)$, the absorbed energy by phosphors that is used for the light conversion $\left(E_{\text {converted }}\right)$ and the heat generated from both Stokes shift $\left(E_{s s}\right)$ and non-radiation $\left(E_{n o n R a d}\right)$.

$$
E_{\text {input }}=E_{\text {transmitted }}+E_{\text {converted }}+E_{\text {ss }}+E_{\text {nonRad }}
$$

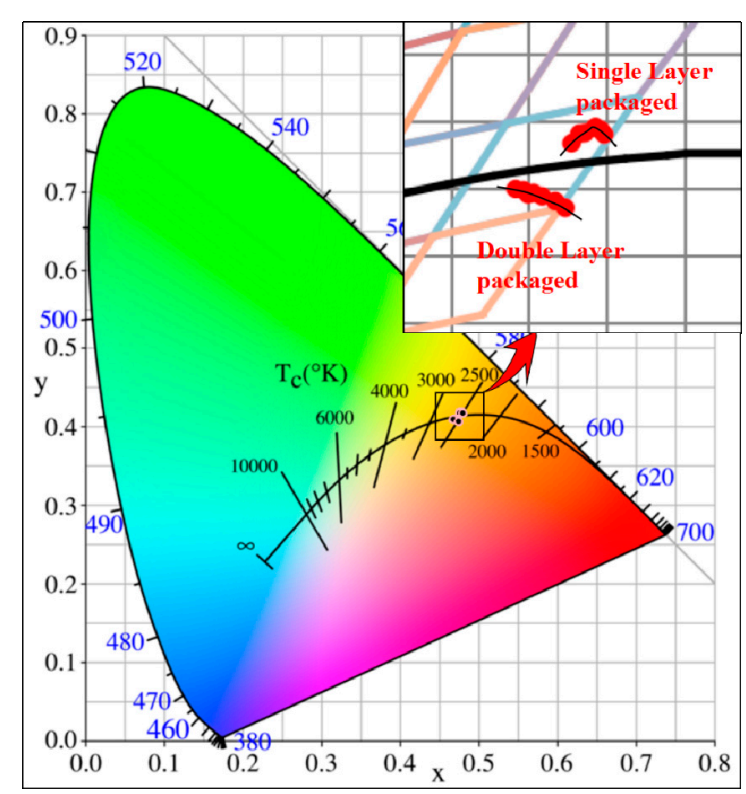

Figure 8. CIE-1931 chromaticity coordinates of traditional dispensing packaged filament and stacked-type packaged filament.

Within the optical tracing process, the light scattering, absorption and conversion are normally considered as dominant effects in most YAG based yellow phosphor models [27]. As shown in Figure 9, light 1 is the blue light emitted by the chip, which is directly emitted through the package. When the blue light emitted by the chip propagates to the green phosphor, it is absorbed and transformed into green light like light 3 , and the propagation direction is isotropic. Light 3 shows that the green light is not absorbed, but directly scattered through the green phosphor when the light fired by one green powder again propagates to another green phosphor. There are very few blue lights absorbed by the green phosphor that can propagate to the red phosphor, as light 4 , that can then reabsorb the photon and turn into red light. The transmission direction of the emitting light of the red phosphor follows the same isotropy. When a blue light emitted by the chip reaches the red phosphor in the film, it is converted into red light like light 5 , and then directly ejected. Some light, like light 6 , does not reach any red phosphor and goes straight through the film. This kind of blue light is only absorbed by the green phosphor and emitted into green light. Light 7 indicates that the emitting light of the red phosphor will not be absorbed when the emitting light of the red phosphor is transmitted to the green phosphor, but instead the scattering phenomenon occurs. Light 8 indicates that blue light is absorbed by the red phosphor and no spectral transformation occurs. This is because the quantum efficiency of the red phosphor is much lower than that of the green phosphor, and the probability of non-radiative recombination is larger. 


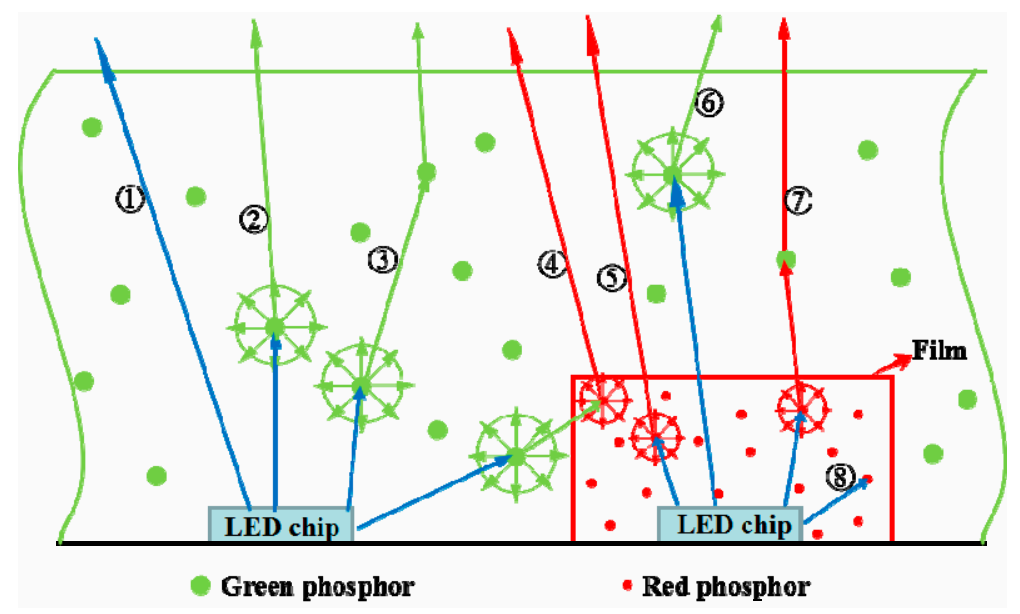

Figure 9. Luminescence mechanism of stacked type packaged filament.

From the schematic diagram, Figure 9, we can clearly see that all light propagates in this special package structure. The excellent luminescence performance of the filament is mainly due to the absorption and transformation of some blue light directly by the green phosphor, which makes up for the defect that the red phosphor in the film blocks the light path, which leads to a reduction in luminous efficiency.

We also found that this special packaging structure can save a large amount of phosphor cost, using a simple calculation. The total mass of all packaging materials used for each traditional dispensing packaged filament was $0.278 \mathrm{~g}$, and the mass fraction of red CASN:Eu ${ }^{2+}$ phosphor and green LuAG:Ce ${ }^{3+}$ phosphor were $3.0 \%$ and $20.89 \%$, respectively. Thus, the masses of red phosphor and green phosphor in each filament were $0.00834 \mathrm{~g}$ and $0.05807 \mathrm{~g}$, respectively. In comparison, in each stacked-type packaged filament, the total mass of the red CASN:Eu ${ }^{2+}$ film was $0.0125 \mathrm{~g}$, in which the mass fraction of red phosphor was $22.2 \%$; meanwhile, the mass of the composition of the silicone matrix was $0.257 \mathrm{~g}$, in which the mass fraction of green LuAG:Ce ${ }^{3+}$ phosphor was $16.67 \%$. As shown in Table 2, the mass of the red CASN:Eu ${ }^{2+}$ phosphor and the green LuAG:Ce ${ }^{3+}$ phosphor on each stacked filament was reduced by $66.7 \%$ and $26.2 \%$, respectively, compared with the traditional packaged filament.

Table 2. Mass of red CASN:Eu ${ }^{2+}$ phosphors and green LuAG:Ce ${ }^{3+}$ phosphors in each filament with different packaging methods.

\begin{tabular}{ccc}
\hline & $\begin{array}{c}\text { Mass of Red CASN:Eu } \\
\text { Phosphor (g) }\end{array}$ & $\begin{array}{c}\text { Mass of Green LuAG:Ce } \\
\text { Phosphor (g) }\end{array}$ \\
\hline Stacked-type filament & 0.00278 & 0.0428 \\
Traditional dispensing filament & 0.00834 & 0.05807 \\
\hline
\end{tabular}

\section{Conclusions}

We have designed an innovative CSP filament with a stacked-type structure and a segmented-type luminescence design, opening a new area in luminous design and providing a better understanding of the tunability of optical properties. A faster and more accurate method to control the luminous parameters of filament has been developed. CCT, CRI and CIE can be set by arranging the proportion of red CSP LEDs and blue chips, or by changing the mass fraction of green phosphors. This fabrication also aimed to solve the spectral overlapping of green and red components for high power phosphor-based LED filaments. The proposed strategy improves luminous efficiency by $7.69 \%$ and $18.97 \%$, more than the traditional dispensing packaged LED filament, when the current is at $30 \mathrm{~mA}$ and $210 \mathrm{~mA}$, respectively, and this reduces the use of red phosphors and green phosphors by $66.7 \%$ and $26.2 \%$, respectively. The protocol for the optical design of stacked-type structure filaments, possessing 
excellent tunability and high luminous efficiency, is an effort to make smarter high-powered LED filament devices.

Author Contributions: Conceptualization, J.Z.; Methodology, M.S.; Software, N.J. and C.Z.; Validation, N.J., W.L. and M.S.; Formal Analysis, N.J.; Investigation, N.J.; Resources, J.L., H.L., Y.L. and X.Y.; Data Curation, J.N.; Writing-Original Draft Preparation, N.J.; Writing-Review \& Editing, N.J.; Visualization, C.Z.; Supervision, J.Z. and B.G.; Project Administration, W.L.; Funding Acquisition, J.Z.

Funding: This work was supported by the Science and Technology Planning Project of Zhejiang Province, China (2018C01046), Enterprise-funded Latitudinal Research Projects (J2016-141), (J2017-171), (J2017-293), (J2017-243).

Conflicts of Interest: The authors declare no conflict of interest.

\section{References}

1. Wang, Z.; Zou, J.; Zhang, C.; Yang, B.; Shi, M.; Li, Y.; Zhou, H.; Liu, Y.; Li, M.; Liu, Z. Facile fabrication and luminescence characteristics of a mixture of phosphors (LuAG:Ce and CaAlSiN3:Eu) in glass for white LED. J. Non-Cryst. Solids 2018, 489, 57-63. [CrossRef]

2. Lee, Y.K.; Lee, J.S.; Heo, J.; Im, W.B.; Chung, W.J. Phosphor in glasses with Pb-free silicate glass powders as robust color-converting materials for white LED applications. Opt. Lett. 2012, 37, 3276. [CrossRef] [PubMed]

3. Lin, Z.; Lin, H.; Xu, J.; Huang, F.; Chen, H.; Wang, B.; Wang, Y. Highly thermal-stable warm w-LED based on Ce:YAG PiG stacked with a red phosphor layer. J. Alloys Compd. 2015, 649, 661-665. [CrossRef]

4. Zhou, J.; Xia, Z. Luminescence color tuning of $\mathrm{Ce}^{3+}, \mathrm{Tb}^{3+}$ and $\mathrm{Eu}^{3+}$ codoped and tri-doped $\mathrm{BaY}_{2} \mathrm{Si}_{3} \mathrm{O}_{10}$ phosphors via energy transfer. J. Mater. Chem. C 2015, 3, 7552-7560. [CrossRef]

5. Schubert, F.E.; Kim, J.K. Solid-state light sources getting smart. Science 2005, 308, 1274-1278. [CrossRef] [PubMed]

6. Gong, M.; Liang, X.; Wang, Y.; Xu, H.; Zhang, L.; Xiang, W. Novel synthesis and optical characterization of phosphor-converted WLED employing Ce:YAG-doped glass. J. Alloys Compd. 2016, 664, 125-132. [CrossRef]

7. Smet, P.F.; Parmentier, A.B.; Poelman, D. Selecting Conversion Phosphors for White Light-Emtting Diodes. J. Electrochem. Soc. 2011, 158, R37-R54. [CrossRef]

8. Pricha, I.; Rossner, W.; Moos, R. Layered Ceramic Phosphors Based on CaAlSiN $3: \mathrm{Eu}^{2+}$ and YAG:Ce ${ }^{3+}$ for White Light-Emitting Diodes. J. Am. Ceram. Soc. 2016, 99, 211-217. [CrossRef]

9. Won, Y.-H.; Jang, H.S.; Cho, K.W.; Song, Y.S.; Jeon, D.Y.; Kwon, H.K. Effect of phosphor geometry on the luminous efficiency of high-power white light-emitting diodes with excellent color rendering property. Opt. Lett. 2009, 34, 1-3. [CrossRef] [PubMed]

10. Chiang, C.-H.; Tsai, H.-Y.; Zhan, T.-S.; Lin, H.-Y.; Fang, Y.-C.; Chu, S.-Y. Effects of phosphor distribution and step-index remote configuration on the performance of white light-emitting diodes. Opt. Lett. 2015, 40, 2830-2833. [CrossRef] [PubMed]

11. Zhu, Y.; Narendran, N. Investigation of Remote-Phosphor White Light-Emitting Diodes with Multi-Phosphor Layers. Jpn. J. Appl. Phys. 2010, 49, 100203. [CrossRef]

12. Lee, J.S.; Arunkumar, P.; Kim, S.; Lee, I.J.; Lee, H.; Im, W.B. Smart design to resolve spectral overlapping of phosphor-in-glass for high-powered remote-type white light-emitting devices. Opt. Lett. 2014, 39, 762-765. [CrossRef] [PubMed]

13. Kim, J.S.; Kwon, O.H.; Jang, J.W.; Lee, S.H.; Han, S.J.; Lee, J.H.; Cho, Y.S. Long-Term Stable, Low-Temperature Remote Silicate Phosphor Thick Films Printed on a Glass Substrate. ACS Comb. Sci. 2015, 17, $234-238$. [CrossRef] [PubMed]

14. Zhu, H.; Che, L.C.; Wenqin, L.; Situan, S.; Zhuguang, L.; Yongsheng, L.; Jintao, K.; En, M.; Yongge, C.; Liu, R.-S.; et al. Highly efficient non-rare-earth red emitting phosphor for warm white light-emitting diodes. Nat. Commun. 2014, 5, 4312. [CrossRef] [PubMed]

15. Zhang, X.; Yu, J.; Wang, J.; Zhu, C.; Zhang, J.; Zou, R.; Lei, B.; Liu, Y.L.; Wu, M. Facile Preparation and Ultra-stable Performance of Single-Component White-Light-Emitting Phosphor-in-Glass used for High-Power Warm White LEDs. ACS Appl. Mater. Interf. 2015, 7, 28122. [CrossRef] [PubMed]

16. Chen, H.; Lin, H.; Xu, J.; Wang, B.; Lin, Z.; Zhou, J.; Wang, Y. Chromaticity-Tunable Phosphor-in-Glass for Long-lifetime High-Power Warm W-LEDs. J. Mater. Chem. C 2015, 3, 8080-8089. [CrossRef] 
17. Xiao, R.; Liang, X.; Li, P.; Di, X.; Xiang, W. A thermally stable warm WLED obtained by screen-printing a red phosphor layer on the LuAG:3+, PiG substrate. Chem. Eng. J. 2016, 306, 858-865.

18. Cai, D.; Yuan, X.; Zhu, D.; Zhou, H.; Li, H.; Zhao, J. Phosphor geometry regulations with separated red and green quantum dot layers for high performance warm white light-emitting diodes. Mater. Res. Bull. 2017, 85, 222-227. [CrossRef]

19. Xiao, H.; Lu, Y.; Xu, Y.; Xue, R. The difference of luminous performance between traditional phosphor packaging LED and remote phosphorLED. Chin. J. Lumin. 2014, 35, 66-72. [CrossRef]

20. Yu, X.; Xie, B.; Chen, Q.; Ma, Y.; Wu, R.; Luo, X. Thermal Remote Phosphor Coating for Phosphor-Converted White-Light-Emitting Diodes. IEEE Trans. Compon. Pack. Manuf. Technol. 2017, 5, 1253-1257. [CrossRef]

21. Kim, H.S.; Machida, K.I.; Horikawa, T.; Hanzawa, H. Luminescence properties of CaAlSiN3:Eu ${ }^{2+}$, phosphor prepared by direct-nitriding method using fine metal hydride powders. J. Alloys Compd. 2015, 633, 97-103. [CrossRef]

22. Yang, L.; Chen, M.; Lv, Z.; Wang, S.; Liu, X.; Liu, S. Preparation of a YAG:Ce phosphor glass by screen-printing technology and its application in LED packaging. Opt. Lett. 2013, 38, 2240-2243. [CrossRef] [PubMed]

23. Zhang, T.; Tang, H.; Li, S.; Wen, Z.; Xiao, X.; Zhang, Y.; Wang, F.; Wang, K. Highly efficient chip scale package (CSP) LED based on surface patterning. IEEE Photon. Technol. Lett. 2016, 29, 1703-17061. [CrossRef]

24. Kim, E.; Unithrattil, S.; Sohn, I.S.; Kim, S.J.; Chung, W.J.; Im, W.B. Facile one-step fabrication of 2-layered and 4-quadrant type phosphor-in-glass plates for white LEDs: An insight into angle dependent luminescence. Opt. Mater. Express 2016, 6, 804-814. [CrossRef]

25. Liu, Y.; Zou, J.; Shi, M.; Li, Y.; Yang, B.; Wang, Z.; Li, W.; Zheng, F.; Zhou, H.; Jiang, N. Effect of phosphor composition and packaging structure of flexible phosphor films on performance of white LEDs. J. Mater. Sci. Mater. Electron. 2018, 29, 18476-18485. [CrossRef]

26. Kang, D.Y.; Wu, E.; Wang, D.M. Modeling white light-emitting diodes with phosphor layers. Appl. Phys. Lett. 2006, 89, 26-822. [CrossRef]

27. Hu, R.; Wang, Y.; Zou, Y.; Chen, X.; Liu, S.; Luo, X. Study on phosphor sedimentation effect in white light-emitting diode packages by modeling multi-layer phosphors with the modified Kubelka-Munk theory. J. Appl. Phys. 2013, 113, 063108. [CrossRef]

(C) 2018 by the authors. Licensee MDPI, Basel, Switzerland. This article is an open access article distributed under the terms and conditions of the Creative Commons Attribution (CC BY) license (http://creativecommons.org/licenses/by/4.0/). 